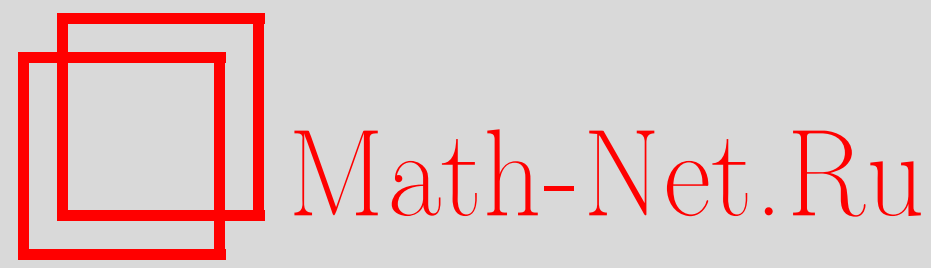

М. Ш. Шабозов, Точные оценки одновременного приближения функций двух переменных и их производных билинейными сплайнами, Матем. заметки, 1996, том 59, выпуск 1, 142-152

DOI: https://doi.org/10.4213/mzm1701

Использование Общероссийского математического портала MathNet.Ru подразумевает, что вы прочитали и согласны с пользовательским соглашением

http: //www . mathnet.ru/rus/agreement

Параметры загрузки:

IP : 54.84 .234 .179

26 апреля 2023 г., 15:31:51

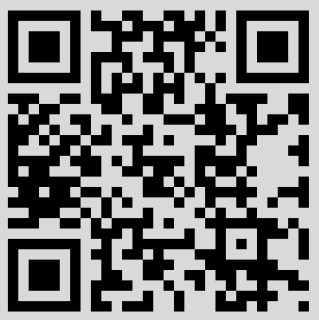


том 59 выпуск 1 январь 1996

\section{ТОЧНЫЕ ОЦЕНКИ ОДНОВРЕМЕННОГО \\ ПРИБЛИЖЕНИЯ ФУНКЦИЙ ДВУХ ПЕРЕМЕННЫХ И ИХ ПРОИЗВОДНЫХ БИЛИНЕЙНЫМИ СПЛАЙНАМИ}

\section{М.Ш. Шабозов}

1. Пусть $f(x, y)$ - непрерывная в области $G=[0,1] \times[0,1]$ функция, Через $C^{(r, s)}(G)$, где $r, s$ - целые неотрицательные числа, обозначим класс функций $f(x, y)$, обладаюших непрерывными частными производными $\partial^{i+j} f / \partial x^{i} \partial y^{j}$, где $i \leqslant r, j \leqslant s$. Далее, при $r=s=0$ полагаем $C^{(0,0)}(G)=$ $C(G)$ с обычной нормой

$$
\|f\|_{C}=\|f\|_{C(G)}=\max \{|f(x, y)|:(x, y) \in G\} .
$$

Специфика двумерного случая позволяет функции $f(x, y) \in C(G)$ сопоставить как полньй модуль непрерьвности [1, с. 335]:

$$
\omega(f ; t, \tau)=\sup \left\{\left|f\left(x^{\prime}, y^{\prime}\right)-f\left(x^{\prime \prime}, y^{\prime \prime}\right)\right|:\left|x^{\prime}-x^{\prime \prime}\right| \leqslant t,\left|y^{\prime}-y^{\prime \prime}\right| \leqslant \tau\right\},
$$

где $\left(x^{\prime}, y^{\prime}\right),\left(x^{\prime \prime}, y^{\prime \prime}\right) \in G$, так и частные модули непрерывности:

$$
\begin{aligned}
& \omega(f ; t, 0)=\sup \left\{\left|f\left(x^{\prime}, y\right)-f\left(x^{\prime \prime}, y\right)\right|:\left|x^{\prime}-x^{\prime \prime}\right| \leqslant t, 0 \leqslant y \leqslant 1\right\}, \\
& \omega(f ; 0, \tau)=\sup \left\{\left|f\left(x, y^{\prime}\right)-f\left(x, y^{\prime \prime}\right)\right|:\left|y^{\prime}-y^{\prime \prime}\right| \leqslant \tau, 0 \leqslant x \leqslant 1\right\},
\end{aligned}
$$

характеризующие изменение $f(x, y)$ вдоль каждой переменной.

Обозначим через $W^{(r, s)} H^{\omega_{1}, \omega_{2}}=W^{(r, s)} H^{\omega_{1}, \omega_{2}}(G)$ класс таких функций $f(x, y) \in C^{(r, s)}(G)$, что для любых двух точек $\left(x^{\prime}, y^{\prime}\right),\left(x^{\prime \prime}, y^{\prime \prime}\right) \in G$

$$
\left|f^{(r, s)}\left(x^{\prime}, y^{\prime}\right)-f^{(r, s)}\left(x^{\prime \prime}, y^{\prime \prime}\right)\right| \leqslant \omega_{1}\left(\left|x^{\prime}-x^{\prime \prime}\right|\right)+\omega_{2}\left(\left|y^{\prime}-y^{\prime \prime}\right|\right),
$$

где $\omega_{1}(t)$ и $\omega_{2}(\tau)$ - заданные модули непрерьвности.

В дальнейшем $r$ и $s$ принимают только значения 0 и 1 . Зададим в области $G$ сетку $\Delta_{m n}=\Delta_{m}^{x} \times \Delta_{n}^{y}$, где $\Delta_{m}^{x}: x_{i}=i / m(i=\overline{0, m}), \Delta_{n}^{y}: y_{j}=j / n$ $(j=\overline{0, n})$. Поставим в соответствие каждой функции $f \in C(G)$ функцию $S_{1,1}(f ; x, y) \in C(G)$, однозначно определенную условиями:

1) на каждой ячейке $G_{i, j}=\left[x_{i-1}, x_{i}\right] \times\left[y_{j-1}, y_{j}\right](i=\overline{1, m}, j=\overline{1, n})$ функция $S_{1,1}(f ; x, y)$ является алгебраическим многочленом первой степени по $x$ и по $y$;

2) $S_{1,1}\left(f ; x_{i}, y_{j}\right)=f\left(x_{i}, y_{j}\right)(i=\overline{0, m}, j=\overline{0, n})$. 
Функции $S_{1,1}(f ; x, y)$ называют интерполяционными сплайнами первой степени двух переменных или билинейными сплайнами (см. [1], [2]).

Для $(x, y) \in G_{i, j}(i=\overline{1, m}, j=\overline{1, n})$ имеет место представление

$$
S_{1,1}(f ; x, y)=\sum_{p=0}^{1} \sum_{k=0}^{1} f\left(x_{i+p-1}, y_{j+k-1}\right) H_{p, i}(x) H_{k, j}(y),
$$

где

$$
\begin{aligned}
& H_{0, i}(x)=m\left(x_{i}-x\right), \quad \sum_{p=0}^{1} H_{p, i}(x) \equiv 1, \\
& H_{0, j}(y)=n\left(y_{j}-y\right), \quad \sum_{k=0}^{1} H_{k, j}(y) \equiv 1 .
\end{aligned}
$$

В дальнейшем полагаем

$$
\begin{aligned}
& F\left(x_{i}, y_{j}\right)=\sum_{p=0}^{1} \sum_{k=0}^{1}(-1)^{p+k} f\left(x_{i+p-1}, y_{j+k-1}\right) \\
& F_{j}\left(x_{i}, y\right)=\sum_{k=0}^{1} \sum_{p=0}^{1}(-1)^{p} f\left(x_{i-p}, y_{j+k-1}\right) H_{k, j}(y), \quad(i=\overline{1, m}, j=\overline{1, n}) . \\
& F_{i}\left(x, y_{j}\right)=\sum_{p=0}^{1} \sum_{k=0}^{1}(-1)^{k} f\left(x_{i+p-1}, y_{j-k}\right) H_{p, i}(x)
\end{aligned}
$$

Ясно, что производные $S_{1,1}^{(r, s)}(f ; x, y)(r, s=0,1,1 \leqslant r+s \leqslant 2)$ на множествах

$$
\begin{array}{ll}
A_{i}=\left\{(x, y): x=x_{i}, 0 \leqslant y \leqslant 1\right\} & (i=\overline{0, m}) \\
B_{j}=\left\{(x, y): y=y_{j}, 0 \leqslant x \leqslant 1\right\} & (j=\overline{0, n})
\end{array}
$$

терпят разрывы. Смешанную производную $S_{1,1}^{(1,1)}(f ; x, y)$, как и в работе [3], доопределим для всех точек $(x, y) \in G_{i, j}^{\prime}=\left[x_{i-1}, x_{i}\right) \times\left[y_{j-1}, y_{j}\right)$ $(i=\overline{1, m-1}, j=\overline{1, n-1})$, полагая $S_{1,1}^{(1,1)}(f ; x, y)=m n F\left(x_{i-1}, y_{j-1}\right)$, а если $(x, y) \in G_{m-1, n-1}=\left[x_{m-1}, x_{m}\right] \times\left[y_{n-1}, y_{n}\right]$, то $S_{1,1}^{(1,1)}(f ; x, y)=$ $m n F\left(x_{m-1}, y_{n-1}\right)$. Положим также: если $(x, y) \in G_{i}^{\prime}=\left[x_{i-1}, x_{i}\right) \times[0,1]$ $(i=\overline{1, m-1})$, то $S_{1,1}^{(1,0)}(f ; x, y)=m F_{j}\left(x_{i-1}, y\right)(j=\overline{1, n})$; если $(x, y) \in$ $G_{m-1}=\left[x_{m-1}, x_{m}\right] \times[0,1]$, то $S_{1,1}^{(1,0)}(f ; x, y)=m F_{j}\left(x_{m-1}, y\right)(j=\overline{1, n})$. Аналогично доопределяется и производная $S_{1,1}^{(1,1)}(f ; x, y)$. 
2. Известно $[1$, гл. 5], что наличие в приближающем подпространстве сплайнов базиса с локальными носителями обуславливает хорошее приближение интерполяционньми сплайнами одновременно и производной интерполируемой функции. В данной работе найдены точные оценки одновременного приближения функций $f(x, y) \in W^{(r, s)} H^{\omega_{1}, \omega_{2}}$ и их производных интерполяционньги билинейньми сплайнами.

Требуется оценить погрешность

$$
\left|e^{(l, q)}(f ; x, y)\right| \stackrel{\text { def }}{=}\left|f^{(l, q)}(x, y)-S_{1,1}^{(l, q)}(f ; x, y)\right| \quad(l, q=0,1)
$$

в каждой точке $(x, y) \in G_{i, j}(i=\overline{1, m}, j=\overline{1, n})$ и для $l \leqslant r, q \leqslant s$, $1 \leqslant r+s \leqslant 2$ найти точное значение величины

$$
\varepsilon^{(l, q)}\left(W^{(r, s)} H^{\omega_{1}, \omega_{2}}\right)=\sup \left\{\left\|e^{(l, q)}(f ; \cdot, \cdot)\right\|_{C}: f \in W^{(r, s)} H^{\omega_{1}, \omega_{2}}\right\} .
$$

Порядковые оценки величины, аналогичной (1), но для иных классов функций имеются в монографиях [2, гл. 2], [4] и [5]. Отметим также, что случай $r=s=0$ рассмотрен в [6], где в предположении вьпуклости $\omega_{1}(t)$ и $\omega_{2}(\tau)$ доказано равенство $\varepsilon\left(H^{\omega_{1}, \omega_{2}}\right)=\omega_{1}(1 / 2 m)+\omega_{2}(1 / 2 n)$, которое является двумерным аналогом результата, полученного в [7].

3. Предварительно докажем одно полезное утверждение.

Лемма. Пусть $f(x, y) \in C(Q)(Q=[a, b] \times[c, d])$ и для любой фиксированной точки $\left(x_{0}, y_{0}\right) \in Q$ выполняются равенства

$$
\int_{a}^{b} f\left(t, y_{0}\right) d t=0,\left(c \leqslant y_{0} \leqslant d\right) ; \quad \int_{c}^{d} f\left(x_{0}, \tau\right) d \tau=0,\left(a \leqslant x_{0} \leqslant b\right) .
$$

Тогда для функиии

$$
F(x, y)=\int_{a}^{x} f\left(t, y_{0}\right) d t+\int_{c}^{y} f\left(x_{0}, \tau\right) d \tau, \quad(x, y) \in Q
$$

справедливы неулучшаемые неравенства

$$
\begin{aligned}
& |F(x, y)| \\
& \quad \leqslant \frac{(b-x)(x-a)}{(b-a)^{2}} \int_{0}^{b-a} \omega(f ; t, 0) d t+\frac{(d-y)(y-c)}{(d-c)^{2}} \int_{0}^{d-c} \omega(f ; 0, \tau) d \tau \\
& \quad \leqslant \frac{(b-x)(x-a)}{b-a} \omega(f ; b-a, 0)+\frac{(d-y)(y-c)}{d-c} \omega(f ; 0, d-c)
\end{aligned}
$$


ДокАЗАТЕЛЬСтво. Зафиксируем $(x, y) \in Q$ и определим функции $\rho(t)$ и $\delta(\tau)$ равенствами

$$
\begin{array}{cl}
g(t)=g(\rho(t)), & a \leqslant t \leqslant x \leqslant \rho(t) \leqslant b, \\
q(\tau)=q(\delta(\tau)), & c \leqslant \tau \leqslant y \leqslant \delta(\tau) \leqslant d,
\end{array}
$$

где функции $g(t)$ и $q(\tau)$ определены соотношениями

$$
\begin{gathered}
g(t)=\int_{a}^{t} \varphi(u) d u, \quad \varphi(u)= \begin{cases}b-x, & a<u<x, \\
a-x, & x<u<b,\end{cases} \\
q(\tau)=\int_{c}^{\tau} \psi(v) d v, \quad \psi(v)= \begin{cases}d-y, & c<v<y, \\
c-y, & y<v<d .\end{cases}
\end{gathered}
$$

В $[8$, c. 95] доказано, что функции $\rho(t)$ и $\delta(\tau)$, определенные в $(5)$ и (6), абсолютно непрерьвны и для них почти всюду вьполняются равенства

$$
\begin{aligned}
g^{\prime}(t) & =g^{\prime}(\rho(t)) \rho^{\prime}(t), \quad a<t<x, \\
q^{\prime}(\tau) & =q^{\prime}(\delta(\tau)) \delta^{\prime}(\tau), \quad c<\tau<y .
\end{aligned}
$$

Из (9) с учетом (7) и (8) следует, что

$$
\rho^{\prime}(t)=\frac{b-x}{a-x}, \quad a<t<x ; \quad \delta^{\prime}(\tau)=\frac{d-y}{c-y}, \quad c<\tau<y .
$$

Используя соотношения (2), запишем очевидное равенство

$$
\begin{aligned}
F(x, y)= & \frac{b-x}{b-a} \int_{a}^{x} f\left(t, y_{0}\right) d t-\frac{x-a}{b-a} \int_{x}^{b} f\left(t, y_{0}\right) d t \\
& +\frac{d-y}{d-c} \int_{c}^{y} f\left(x_{0}, \tau\right) d \tau-\frac{y-c}{d-c} \int_{y}^{d} f\left(x_{0}, \tau\right) d \tau .
\end{aligned}
$$

Сделав замену $t=\rho(u)$ и $\tau=\delta(v)$ соответственно в промежутках $[x, b]$ и $[y, d]$, имеем:

$$
\begin{aligned}
|F(x, y)| \leqslant & \frac{b-x}{b-a} \int_{a}^{x}\left|f\left(t, y_{0}\right)-f\left(\rho(t), y_{0}\right)\right| d t \\
& +\frac{d-y}{d-c} \int_{c}^{y}\left|f\left(x_{0}, \tau\right)-f\left(x_{0}, \delta(\tau)\right)\right| d \tau \\
\leqslant & \frac{b-x}{b-a} \int_{a}^{x} \omega(f ; \rho(t)-t, 0) d t+\frac{d-y}{d-c} \int_{c}^{y} \omega(f ; 0, \delta(\tau)-\tau) d \tau \\
= & \frac{(b-x)(x-a)}{(b-a)^{2}} \int_{0}^{b-a} \omega(f ; t, 0) d t
\end{aligned}
$$




$$
\begin{aligned}
& +\frac{(d-y)(y-c)}{(d-c)^{2}} \int_{0}^{d-c} \omega(f ; 0, \tau) d \tau \\
\leqslant & \frac{(b-x)(x-a)}{b-a} \omega(f ; b-a, 0)+\frac{(d-y)(y-c)}{d-c} \omega(f ; 0, d-c) .
\end{aligned}
$$

На функции $f(x, y)=g_{h}(x)+q_{\eta}(y)$ проверяется неулучшаемость неравенств (4) (при малых $h$ и $\eta$ ), где $g_{h}(x)$ и $q_{\eta}(y)$ - функции Стеклова для заданных равенствами $(7)$ и (8) функций $g(x)$ и $q(y)$. Лемма доказана.

Если обозначить через $\omega\left(f ;\left[t_{i-1}, t_{i}\right], t, 0\right)$ и $\omega\left(f ; 0,\left[\tau_{j-1}, \tau_{j}\right], \tau\right)$ - частные модули непрерывности функции $f(t, \tau) \in C(G)$, рассматриваемые только на ячейке $G_{i, j}=\left[t_{i-1}, t_{i}\right] \times\left[\tau_{j-1}, \tau_{j}\right](i=\overline{1, m}, j=\overline{1, n})$, то из доказанной леммы вытекает следующее утверждение.

ПРЕДЛОЖЕНИЕ. Пусть $f(t, \tau) \in C^{(1,0)}(G) \cap C^{(0,1)}(G) u S_{1,1}(f ; t, \tau)-$ билинейный сплайн, интерполирующий функиию $f(t, \tau)$ в узлах $\left(t_{i}, \tau_{j}\right)$ $(i=\overline{0, m}, j=\overline{0, n})$ произвольного разбиения $G_{i, j}=\left[t_{i-1}, t_{i}\right] \times\left[\tau_{j-1}, \tau_{j}\right]$ $(i=\overline{1, m}, j=\overline{1, n})$ квадрата $G$. Тогда в каждой точке $(x, y) \in G_{i, j}$ выполняются неравенства

$$
\begin{aligned}
& \left|f(x, y)-S_{1,1}(f ; x, y)\right| \\
& \leqslant \frac{\left(t_{i}-x\right)\left(x-t_{i-1}\right)}{\left(t_{i}-t_{i-1}\right)^{2}} \int_{0}^{t_{i}-t_{i-1}} \omega\left(f^{(1,0)} ;\left[t_{i-1}, t_{i}\right], t, 0\right) d t \\
& \quad+\frac{\left(\tau_{j}-y\right)\left(y-\tau_{j-1}\right)}{\left(\tau_{j}-\tau_{j-1}\right)^{2}} \int_{0}^{\tau_{j}-\tau_{j-1}} \omega\left(f^{(0,1)} ; 0,\left[\tau_{j-1}, \tau_{j}\right], \tau\right) d \tau \\
& \leqslant \frac{\left(t_{i}-x\right)\left(x-t_{i-1}\right)}{t_{i}-t_{i-1}} \omega\left(f^{(1,0)} ;\left[t_{i-1}, t_{i}\right], t_{i}-t_{i-1}, 0\right) \\
& \quad+\frac{\left(\tau_{j}-y\right)\left(y-\tau_{j-1}\right)}{\tau_{j}-\tau_{j-1}} \omega\left(f^{(0,1)} ; 0,\left[\tau_{j-1}, \tau_{j}\right], \tau_{j}-\tau_{j-1}\right),
\end{aligned}
$$

неулучиаемые на всем множестве $C^{(1,0)}(G) \cap C^{(0,1)}(G)$.

Положим

$$
\begin{aligned}
\Omega\left(\omega_{1}, \omega_{2}\right)=\{f: f \in & C^{(1,0)}(G) \cap C^{(0,1)}(G), \\
& \left.\omega\left(f^{(1,0)} ; t, 0\right) \leqslant \omega_{1}(t), \omega\left(f^{(0,1)} ; 0, \tau\right) \leqslant \omega_{2}(\tau)\right\} .
\end{aligned}
$$

Тогда при равномерном разбиении $\left(x_{i}, y_{j}\right)=(i / m, j / n)(i=\overline{0, m}, j=\overline{0, n})$ в силу доказанной леммы для функций $f(x, y) \in \Omega\left(\omega_{1}, \omega_{2}\right)$ в точке $(x, y) \in$ 
$\left\{x_{i-1}<x<x_{i}, y_{j-1}<y<y_{j}\right\}$ справедлива оценка

$$
\begin{aligned}
\left|f(x, y)-S_{1,1}(f ; x, y)\right| \leqslant & m^{2}\left(x_{i}-x\right)\left(x-x_{i-1}\right) \int_{0}^{1 / m} \omega_{1}(t) d t \\
& +n^{2}\left(y_{j}-y\right)\left(y-y_{j-1}\right) \int_{0}^{1 / n} \omega_{2}(\tau) d \tau \\
= & H_{0, i}(x) H_{1, i}(x) \int_{0}^{1 / m} \omega_{1}(t) d t \\
& +H_{0, j}(y) H_{1, j}(y) \int_{0}^{1 / n} \omega_{2}(\tau) d \tau .
\end{aligned}
$$

Из неравенства (10) сразу вытекает

ТЕОРЕМА 1. Пусть $\omega_{1}(t) u \omega_{2}(\tau)$ - произвольные выпуклые модули непрерывности. Тогда для любых $m$ и $n$ справедливо равенство

$$
\begin{aligned}
\varepsilon\left(\Omega\left(\omega_{1}, \omega_{2}\right)\right) & \stackrel{\text { def }}{=} \sup \left\{\|e(f ; \cdot, \cdot)\|_{C}: f \in \Omega\left(\omega_{1}, \omega_{2}\right)\right\} \\
& =\frac{1}{4} \int_{0}^{1 / m} \omega_{1}(t) d t+\frac{1}{4} \int_{0}^{1 / n} \omega_{2}(\tau) d \tau
\end{aligned}
$$

Если же $\omega_{1}(t)$ и $\omega_{2}(\tau)$ - произвольные модули непрерывности, то

$$
\varepsilon\left(\Omega\left(\omega_{1}, \omega_{2}\right)\right)=\frac{\theta_{\omega}}{4} \int_{0}^{1 / m} \omega_{1}(t) d t+\frac{\theta_{\omega}}{4} \int_{0}^{1 / n} \omega_{2}(\tau) d \tau \quad\left(2 / 3 \leqslant \theta_{\omega} \leqslant 1\right) .
$$

ДокаЗАТЕЛЬСТво. Так как

$$
\begin{aligned}
\max \left\{H_{0, i}(x) H_{1, i}(x): x\right. & \left.\in\left[x_{i-1}, x_{i}\right]\right\} \\
& =\max \left\{H_{0, j}(y) H_{1, j}(y): y \in\left[y_{j-1}, y_{j}\right]\right\}=\frac{1}{4}
\end{aligned}
$$

то из $(11)$ для любого $(x, y) \in G_{i, j}$ имеем

$$
\left|e_{i, j}(f ; x, y)\right| \leqslant \frac{1}{4} \int_{0}^{1 / m} \omega_{1}(t) d t+\frac{1}{4} \int_{0}^{1 / n} \omega_{2}(\tau) d \tau
$$

Следовательно,

$$
\varepsilon\left(\Omega\left(\omega_{1}, \omega_{2}\right)\right) \leqslant \frac{1}{4} \int_{0}^{1 / m} \omega_{1}(t) d t+\frac{1}{4} \int_{0}^{1 / n} \omega_{2}(\tau) d \tau
$$

До сих пор на $\omega_{1}(t)$ и $\omega_{2}(\tau)$ не накладьвалось никаких ограничений. Считая теперь $\omega_{1}(t)$ и $\omega_{2}(\tau)$ вьпуклыми модулями непрерьвности, докажем, 
что в (13) имеет место знак равенства. С этой целью зададим соответственно на $[0,1 / m]$ и $[0,1 / n]$ две функции:

$$
\begin{aligned}
& \varphi_{0}(t)=\left\{\begin{aligned}
\frac{1}{2} \omega_{1}\left(\frac{1}{m}-2 t\right), & 0 \leqslant t \leqslant \frac{1}{2 m}, \\
-\frac{1}{2} \omega_{1}\left(2 t-\frac{1}{m}\right), & \frac{1}{2 m} \leqslant t \leqslant \frac{1}{m} ;
\end{aligned}\right. \\
& \psi_{0}(\tau)=\left\{\begin{aligned}
\frac{1}{2} \omega_{2}\left(\frac{1}{n}-2 \tau\right), & 0 \leqslant \tau \leqslant \frac{1}{2 n}, \\
-\frac{1}{2} \omega_{2}\left(2 \tau-\frac{1}{n}\right), & \frac{1}{2 n} \leqslant \tau \leqslant \frac{1}{n} .
\end{aligned}\right.
\end{aligned}
$$

Для $t \in[1 / m, 1 / 2 m]$ положим $\varphi_{0}(t)=\varphi_{0}(2 / m-t)$ и продолжим функцию $\varphi_{0}(t)$ периодически с периодом $2 / m$ на всю ось. Аналогично с периодом $2 / n$ продолжим на всю ось функцию $\psi_{0}(\tau)$. Наконец, положим

$$
f_{0}(x, y)=\int_{0}^{x} \varphi_{0}(t) d t+\int_{0}^{y} \psi_{0}(\tau) d \tau, \quad(x, y) \in G .
$$

Очевидно, что $f_{0} \in \Omega\left(\omega_{1}, \omega_{2}\right)$. Кроме того

$$
\begin{aligned}
& \left\|f_{0}(\cdot, \cdot)-S_{1,1}\left(f_{0} ; \cdot, \cdot\right)\right\|_{C}=\left\|f_{0}(\cdot, \cdot)\right\|_{C} \\
& \quad=\frac{1}{2} \int_{0}^{1 / 2 m} \omega_{1}\left(\frac{1}{m}-2 t\right) d t+\frac{1}{2} \int_{0}^{1 / 2 n} \omega_{2}\left(\frac{1}{n}-2 \tau\right) d \tau \\
& \quad=\frac{1}{4} \int_{0}^{1 / m} \omega_{1}(t) d t+\frac{1}{4} \int_{0}^{1 / n} \omega_{2}(\tau) d \tau .
\end{aligned}
$$

Таким образом доказано, что для выпуклых модулей непрерывности $\omega_{1}(t)$ и $\omega_{2}(\tau)$ неравенство $(13)$ обращается в равенство. Если же $\omega_{1}(t)$ и $\omega_{2}(\tau)-$ произвольные модули непрерывности, то будем полагать $\varphi_{1}(t)=\frac{2}{3} \varphi_{0}(t)$, $\psi_{1}(\tau)=\frac{2}{3} \psi_{0}(\tau)$. Тогда функция

$$
f_{1}(x, y)=\int_{0}^{x} \varphi_{1}(t) d t+\int_{0}^{y} \psi_{1}(\tau) d \tau, \quad(x, y) \in G,
$$

принадлежит классу $\Omega\left(\omega_{1}, \omega_{2}\right)[9$, c. 24$]$. Однако в этом случае

$$
\begin{aligned}
\varepsilon\left(\Omega\left(\omega_{1}, \omega_{2}\right)\right) & \geqslant\left\|f_{1}(\cdot, \cdot)-S_{1,1}\left(f_{1} ; \cdot, \cdot\right)\right\|_{C}=\left\|f_{1}(\cdot, \cdot)\right\|_{C} \\
& =\frac{2}{3}\left(\frac{1}{4} \int_{0}^{1 / m} \omega_{1}(t) d t+\frac{1}{4} \int_{0}^{1 / n} \omega_{2}(\tau) d \tau\right) .
\end{aligned}
$$

Из (13) и (14) следует (12), чем и завершается доказательство теоремы 1.

Сопоставляя неравенства (13) и (14), получаем

СлЕДСТВИЕ. Для любых модулей непрерывности $\omega_{1}(t) u \omega_{2}(\tau)$ справедливо неравенство

$$
\frac{2}{3} \mathscr{I}_{m, n}\left(\omega_{1}, \omega_{2}\right) \leqslant \varepsilon\left(\Omega\left(\omega_{1}, \omega_{2}\right)\right) \leqslant \mathscr{I}_{m, n}\left(\omega_{1}, \omega_{2}\right),
$$

əде

$$
\mathscr{I}_{m, n}\left(\omega_{1}, \omega_{2}\right)=\frac{1}{4} \int_{0}^{1 / m} \omega_{1}(t) d t+\frac{1}{4} \int_{0}^{1 / n} \omega_{2}(\tau) d \tau .
$$


ТЕорема 2. Пусть $\omega_{1}(t) u \omega_{2}(\tau)$ - произвольные модули непрерывности. Тогда для любых $m$ и $n$ имеет место равенство

$$
\varepsilon^{(1,1)}\left(W^{(1,1)} H^{\omega_{1}, \omega_{2}}\right)=m \int_{0}^{1 / m} \omega_{1}(t) d t+n \int_{0}^{1 / n} \omega_{2}(\tau) d \tau .
$$

ДокАЗАТЕЛЬСтво. Воспользуемся интегральным представлением погрешности, полученным в [3],

$$
\begin{aligned}
e_{i, j}^{(1,1)}(f ; x, y)= & f^{(1,1)}(x, y)-S_{1,1}^{(1,1)}(f ; x, y) \\
= & \sum_{p=0}^{1} \sum_{k=0}^{1} H_{p, i}(x) H_{k, j}(y) \int_{0}^{1} \int_{0}^{1}\left[f^{(1,1)}(x, y)\right. \\
& \left.-f^{(1,1)}\left(x+t\left(x_{i+p-1}-x\right), y+\tau\left(y_{j+k-1}-y\right)\right)\right] d t d \tau \\
& (x, y) \in G_{i, j} \quad(i=\overline{1, m}, j=\overline{1, n}) .
\end{aligned}
$$

Оценивая равенство (15) по абсолютной величине и имея ввиду, что $f(x, y) \in W^{(1,1)} H^{\omega_{1}, \omega_{2}}$, имеем

$$
\left|e_{i, j}^{(1,1)}(f ; x, y)\right| \leqslant \Phi_{i, j}(x, y), \quad(x, y) \in G_{i, j} \quad(i=\overline{1, m}, j=\overline{1, n}),
$$

где

$$
\Phi_{i, j}(x, y)=m \sum_{p=0}^{1} \int_{0}^{m^{-1} H_{p, i}(x)} \omega_{1}(t) d t+n \sum_{k=0}^{1} \int_{0}^{n^{-1} H_{k, j}(y)} \omega_{2}(\tau) d \tau .
$$

Очевидно, что

$$
\max \left\{\Phi_{i, j}(x, y):(x, y) \in G_{i, j}\right\}=m \int_{0}^{1 / m} \omega_{1}(t) d t+n \int_{0}^{1 / n} \omega_{2}(\tau) d \tau .
$$

В силу (16) и (17) запишем оценку сверху

$$
\varepsilon^{(1,1)}\left(W^{(1,1)} H^{\omega_{1}, \omega_{2}}\right) \leqslant m \int_{0}^{1 / m} \omega_{1}(t) d t+n \int_{0}^{1 / n} \omega_{2}(\tau) d \tau .
$$

\section{Положим}

$$
f_{2}(x, y)=y \int_{0}^{x} \varphi_{2}(t) d t+x \int_{0}^{y} \psi_{2}(\tau) d \tau, \quad(x, y) \in G,
$$

где

$$
\begin{array}{r}
\varphi_{2}(x)=\omega_{1}\left((-1)^{i}(1 / m-x)\right)-m \int_{0}^{1 / m} \omega_{1}(t) d t, \\
x \in[i / m,(i+1) / m] \quad(i=0,1), \\
\psi_{2}(y)=\omega_{2}\left((-1)^{j}(1 / n-y)\right)-n \int_{0}^{1 / n} \omega_{2}(\tau) d \tau, \\
y \in[j / n,(j+1) / n] \quad(j=0,1), \\
\varphi_{2}(x+2 / m)=\varphi_{2}(x), \quad \psi_{2}(y+2 / n)=\psi_{2}(y) .
\end{array}
$$


Ясно, что функция $f_{2}(x, y) \in W^{(1,1)} H^{\omega_{1}, \omega_{2}}$. Непосредственной проверкой убеждаемся, что

$$
\left\|e^{(1,1)}\left(f_{2} ; \cdot, \cdot\right)\right\|_{C}=\left\|f_{2}^{(1,1)}(\cdot, \cdot)\right\|_{C}=m \int_{0}^{1 / m} \omega_{1}(t) d t+n \int_{0}^{1 / n} \omega_{2}(\tau) d \tau .
$$

Теорема 2 доказана.

Теоремы 1 и 2 в некотором смысле являются двумерными аналогами основных результатов работ [7] и [8]. Рассмотрим еще вопрос о приближении частных производных $f^{(1,0)}(x, y)$ и $f^{(0,1)}(x, y)$ соответствующими производньми $S_{1,1}^{(1,0)}(f ; x, y), S_{1,1}^{(0,1)}(f ; x, y)$ билинейного сплайна.

ТЕорема 3. Пусть $\omega_{1}(t)-$ произвольный, $а \omega_{2}(\tau)-$ выпуклый модули непрерывности. Тогда имеет место равенство

$$
\varepsilon^{(1,0)}\left(W^{(1,0)} H^{\omega_{1}, \omega_{2}}\right)=m \int_{0}^{1 / m} \omega_{1}(t) d t+\omega_{2}(1 / 2 n) .
$$

Если же $\omega_{1}(t)$ - выпуклый, $\omega_{2}(\tau)$ - произвольный модули непрерывности, то

$$
\varepsilon^{(0,1)}\left(W^{(0,1)} H^{\omega_{1}, \omega_{2}}\right)=\omega_{1}(1 / 2 m)+n \int_{0}^{1 / n} \omega_{2}(\tau) d \tau .
$$

ДоказАТЕЛЬСтво. Равенства (19) и (20) доказываются одинаково, поэтому докажем только (19). Используя определение и свойства разделенных разностей функции $f(x, y)$ (см., например, [11]), находим

$$
\begin{gathered}
e_{i, j}^{(1,0)}(f ; x, y)=f^{(1,0)}(x, y)-S_{1,1}^{(1,0)}(f ; x, y)=f^{(1,0)}(x, y)-m F_{j}\left(x_{i}, y\right) \\
=f^{(1,0)}(x, y)-m \sum_{k=0}^{1} H_{k, j}(y)\left[f\left(x_{i}, y_{j+k-1}\right)-f\left(x_{i-1}, y_{k+j-1}\right)\right] \\
=f^{(1,0)}(x, y)-\sum_{p=0}^{1} \sum_{k=0}^{1} H_{p, i}(x) H_{k, j}(y) f\left(x_{i+p-1}, x ; y_{j+k-1}\right), \\
(x, y) \in G_{i, j}(i=\overline{1, m}, j=\overline{1, n}) .
\end{gathered}
$$

Представляя разделенно-разностные отношения в интегральной форме, перепишем (21) в следующем виде

$$
\begin{aligned}
e_{i, j}^{(1,0)}(f ; x, y) & =\sum_{p=0}^{1} \sum_{k=0}^{1} H_{p, i}(x) H_{k, j}(y) \\
& \times \int_{0}^{1}\left[f^{(1,0)}(x, y)-f^{(1,0)}\left(x+t\left(x_{i+p-1}-x\right) ; y_{j+k-1}\right)\right] d t .
\end{aligned}
$$


Отсюда в силу принадлежности $f(x, y)$ классу $W^{(1,0)} H^{\omega_{1}, \omega_{2}}$ имеем

$$
\begin{aligned}
\left|e_{i, j}^{(1,1)}(f ; x, y)\right| \leqslant & m \sum_{p=0}^{1} \int_{0}^{m^{-1} H_{p, i}(x)} \omega_{1}(t) d t \\
& +\sum_{k=0}^{1} H_{k, j}(y) \omega_{2}\left(\left|y-y_{j+k-1}\right|\right) \equiv \Phi(x)+F(y) .
\end{aligned}
$$

Непосредственным вычислением находим:

$$
\max \left\{\Phi(x): x \in\left[x_{i-1}, x_{i}\right]\right\}=m \int_{0}^{1 / m} \omega_{1}(t) d t .
$$

В силу вьпуклости $\omega_{2}(\tau)$ и тождества $\sum_{k=0}^{1} H_{k, j}(y) \equiv 1$, для функции $F(y)$ имеет место неравенство

$$
\begin{aligned}
F(y) & =\sum_{k=0}^{1} H_{k, j}(y) \omega_{2}\left(\left|y-y_{j+k-1}\right|\right) \\
& =H_{0, j}(y) \omega_{2}\left(y-y_{j-1}\right)+H_{1, j}(y) \omega_{2}\left(y_{j}-y\right) \\
& \leqslant \omega_{2}\left[H_{0, j}(y)\left(y-y_{j-1}\right)+H_{1, j}(y)\left(y_{j}-y\right)\right] .
\end{aligned}
$$

Но для функции $\lambda(y)=H_{0, j}(y)\left(y-y_{j-1}\right)+H_{1, j}(y)\left(y_{j}-y\right)$,

$$
\max \left\{\lambda(y): y \in\left[y_{j-1}, y_{j}\right]\right\}=\lambda\left(\frac{y_{j-1}+y_{j}}{2}\right)=\frac{1}{2 n},
$$

а потому из (24) следует, что

$$
\max \left\{F(y): y \in\left[y_{j-1}, y_{j}\right]\right\} \leqslant \omega_{2}(1 / 2 n) .
$$

В качестве экстремальной рассмотрим функцию

$$
f_{3}(x, y)=\int_{0}^{x} \varphi_{2}(t) d t+x \psi_{3}(y), \quad(x, y) \in G,
$$

где $\varphi_{2}(x)$ - функция, определенная равенством $(18)$, а $\psi_{3}(y)$ определяется следуюшим образом:

$$
\psi_{3}(y)=\left\{\begin{array}{ll}
\omega_{2}\left(y-y_{j-1}\right), & y_{j-1} \leqslant y \leqslant y_{j-1}+\frac{1}{2 n}, \\
\omega_{2}\left(y_{j}-y\right), & y_{j-1}+\frac{1}{2 n} \leqslant y \leqslant y_{j},
\end{array} \quad(j=\overline{1, n}) .\right.
$$

Легко проверить, что $f_{3}(x, y) \in W^{(1,0)} H^{\omega_{1}, \omega_{2}}$, и так как

$$
\left\|e^{(1,0)}\left(f_{3} ; \cdot, \cdot\right)\right\|_{C}=\left\|f_{3}^{(1,0)}(\cdot, \cdot)\right\|_{C}=m \int_{0}^{1 / m} \omega_{1}(t) d t+\omega_{2}(1 / 2 n),
$$

то равенство (19) и вместе с ним теорема 3 доказаны.

Автор благодарен Н. П. Корнейчуку за консультации и полезное обсуждение работы. 


\section{СПИСОК ЦИТИРОВАННОЙ ЛИТЕРАТУРЫ}

[1] Корнейчук Н. П. Сплайны в теории приближения. М.: Наука, 1984.

[2] Завьялов Ю. С., Квасов Б. И., Мирошниченко В. Л. Методы сплайнфункций. М.: Наука, 1980.

[3] Вакарчук С. Б. К интерполяции билинейньми сплайнами // Матем. заметки. 1990. Т. 47. № 5. С. 26-29.

[4] Алберг Дж., Нильсон Э., Уолш Дж. Теория сплайнов и ее приложения. М.: Мир, 1972.

[5] Стечкин С. Б., Субботин Ю.Н. Сплайны в вычислительной математике. М.: Наука, 1976.

[6] Сторчай В.Ф. Приближение функций двух переменных многогранными функциями в равномерной метрике // Изв. вузов. Матем. 1973. № 8. С. 84-88.

[7] Малозёмов В.Н. Об отклонении ломаных // Вестн. ЛГУ. Матем., мех., астрон. 1966. № 7. С. 150-153.

[8] Корнейчук Н. П. Экстремальные задачи теории приближения. М.: Наука, 1976.

[9] Степанец А. И. Равномерные приближения тригонометрическими полиномами. Киев: Наукова думка, 1981.

[10] Малозёмов В. Н. К полигональной интерполяции // Матем. заметки. 1967. T. 1. № 5. C. 537-540.

[11] Микеладзе Ш. Е. Численные методы математического анализа. М.: Гостехиздат, 1953. 